\title{
STUDIES ON THE ALKYLATION OF QUINOLIN-2(1H)-ONE DERIVATIVES
}

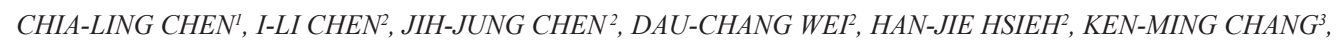 \\ CHERNG-CHYI TZENG ${ }^{3}$ AND TAI-CHI WANG ${ }^{2 *}$ \\ ${ }^{1}$ Department of Pharmacy, KUO General Hospital, Tainan City 700, Taiwan \\ ${ }^{2}$ Department of Pharmacy, Tajen University, Pingtung 907, Taiwan \\ ${ }^{3}$ Departament of Medicinal and Applied Chemistry, College of Life Science, Kaohsiung Medical University, Kaohsiung 807, Taiwan
}

\begin{abstract}
Alkylation of quinolin-2(1H)-one (1) and its $\mathrm{C}(6)$ and $\mathrm{C}(7)$ substituted dervatives $(\mathrm{OMe}, \mathrm{OBn}$, and $\mathrm{Cl}$ ) with 2-bromoacetophenone or chloroacetone under basic condition $\left(\mathrm{K}_{2} \mathrm{CO}_{3}\right.$ in DMF) gave a mixture of $\mathrm{N}_{1}$ - and $\mathrm{O}_{2}$ - alkylated products with the former one as a major product. However, alkylation of 8-methoxy-, 8-benzyloxy-, and 8-chloro- quinolin-2 $(1 H)$-ones under the same reaction conditions gave exclusively $\mathrm{O}_{2}$-alkylated products.
\end{abstract}

Keywords: N-Alkylation; O-Alkylation; Quinolin-2(1H)-one.

\section{INTRODUCTION}

Quinolin-2(1H)-one (carbostyril) skeleton is present in a large number of biologically active compounds which exhibit antiplatelet, anti-inflammatory, anti-ulcer, vasodilatory, and phosphodiesterase inhibitory activities ${ }^{1-12}$ Carteolol, for example, has been used clinically as a $\beta$-adrenergic blocking agent ${ }^{8}$. Over the past few years, we were particularly interested in the synthesis of $\alpha$-methylene- $\gamma$-butyrolactones bearing heterocycles such as coumarins, flavones, xanthones, quinolines, and quinolin- $2(1 H)$-ones and the evaluation of their cardiovascular and cytotoxic activities ${ }^{9-14}$. Among these heterocycles, coumarins exhibited the most potent inhibitory activities on the high- $\mathrm{K}^{+}$medium, $\mathrm{Ca}^{2+}$-induced vasoconstriction, and the norepinephrine-induced phasic and tonic vasoconstrictions, while quinolin-2(1H)-ones proved to be the most active against platelet aggregation. A number of quinolin-2 $(1 H)$-one $\alpha$-methylene- $\gamma$-butyrolactones were found to exhibit potent antiproliferative activities $^{14}$.

These biologically active quinolin-2(1H)-one derivatives were synthesized from their hydroxyl precursors by alkylation and the Reformatsky-type condensation. The Reformatsky-type condensation is quite straightforward while the alkylation of quinolin-2(1H)-one involves a competitive alkylation leading to the mixture of $\mathrm{N}_{1}$ - and $\mathrm{O}_{2}$ - alkylated products. Although it is well known that alkylation of 2-pyridones gave a mixture of both $\mathrm{N}$ - and $\mathrm{O}$ - alkylated products, ${ }^{15-17}$ relatively few systematic studies of this phenomenon have been published. Hopkins et $a l^{18}$ demonstrated that alkylation of 2-pyridone with benzyl chloride and sodium salt in DMF occurred at $N_{1}$-position whereas using the silver salt in benzene afforded exclusively the $O_{2}$-alkylated product. The Mitsunobu reaction has also been applied to alkylate 2-pyridones to discover the relationship between the ratio of $\mathrm{N}$ - and O- alkylation products. ${ }^{19,20}$

The alkylation of C-5 substituted 2-phenyl-4-quinolones was also investigated and showed that the ratio of $N$-alkylation versus $\mathrm{O}$-alkylation is highly dependent on the property of C-5 group. Both $\mathrm{N}$-alkylation and O-alkylation products were detected for $\mathrm{C}-5$ methoxy derivative while the sole product of $\mathrm{N}$-alkylation was obtained for the C-5 hydroxy derivative. The alkylation of these 2-phenyl-4-quinolones was conducted under several conditions (NaH/THF, $\mathrm{NaH} / \mathrm{DMF}, \mathrm{K}_{2} \mathrm{CO}_{3}$ /acetone, $\left.\mathrm{K}_{2} \mathrm{CO}_{3} / \mathrm{DMF}\right)$ at different temperatures. The $\mathrm{K}_{2} \mathrm{CO}_{3} / \mathrm{DMF}$ system and temperature elevation led to the highest yields but there was no significant influence on the regioselectivity compared to the other systems used. ${ }^{21}$ Studies on N-alkylation versus O-alkylation in various ambident heterocyclic compounds under phase transfer conditions have also been reported..$^{22-25}$ We have obtained a mixture of $\mathrm{N}_{1}$ - and $\mathrm{O}_{2}$ - alkylated products from the alkylation of quinolin-2(1H)one (1) with $\mathrm{N}_{1}$-alkylation as the major product (Scheme 1$)^{10}$. Alkylation of 6-acetoxyquinolin-2 $(1 H)$-one $(2)$ gave a mixture of $\mathrm{N}_{1}$ - and $\mathrm{O}_{2}$ - alkylated products with $\mathrm{N}_{1}$-alkylation as the major product ${ }^{11}$. However, a sole $\mathrm{O}_{2}$ alkylated product was obtained from the alkylation of 8 -acetoxyquinolin2(1H)-one (9) with 2-bromoacetophenone and potassium carbonate in $\mathrm{DMF}^{12}$. Guo et. al. have also reported that the alkylation of 8-hydroxyquinolin-2(1H)one with 3-methoxybenzyl bromide under basic condition to afford a mixture of $\mathrm{N}_{1}$ - and $\mathrm{O}_{2}$ - alkylated products with the former one as a major product ${ }^{26}$. Alkylation of 8-hydroxyquinolin-2(1H)-one was similar to 2 but was distincted from 9 prompted us to explore the alkylation of various quinolin-2 $(1 H)$-one derivatives. The steric and electronic effects of different substituents which influence the $\mathrm{N}_{1}$ - and $\mathrm{O}_{2}$ - alkylation of quinolin-2 $(1 \mathrm{H})$-ones are described.

\section{RESULTS AND DISCUSSION}

Alkylation of quinolin-2(1H)-one (1) with 2-bromoacetophenone and potassium carbonate in DMF gave a mixture of $\mathrm{N}_{1}-$ and $\mathrm{O}_{2}$ - alkylated products (13a and 13b respectively) with 13a as a major product (Scheme 1) but not a sole product as our previous report ${ }^{10}$. The structure of 13a was confirmed by the long-range ${ }^{1} \mathrm{H},{ }^{13} \mathrm{C}$-HETCOR experiment in which $\mathrm{N}(1)-\mathrm{CH}_{2}(5.81 \mathrm{ppm})$ was coupled to C-atoms with resonances of $192.37\left({ }^{2} \mathrm{~J}\right), 162.11^{2}\left({ }^{3} \mathrm{~J}\right), 139.55\left({ }^{3} \mathrm{~J}\right)$, and $48.68\left({ }^{1} J\right) \mathrm{ppm}$ corresponding to $\mathrm{C}\left(2^{\prime}\right), \mathrm{C}(2), \mathrm{C}(8 \mathrm{a})$, and $\mathrm{C}\left(1^{\prime}\right)$ respectively. Structure of $\mathbf{1 3 b}$ was also confirmed by ${ }^{1} \mathrm{H},{ }^{13} \mathrm{C}$-HETCOR experiment in which $\mathrm{O}(2)-\mathrm{CH}_{2}(5.76 \mathrm{ppm})$ was coupled to C-atoms with resonances of 194.49 $\left({ }^{2} J\right), 160.68\left({ }^{3} J\right)$, and $67.48\left({ }^{1} J\right) \mathrm{ppm}$ corresponding to $\mathrm{C}\left(2^{\prime}\right), \mathrm{C}(2)$, and $\mathrm{C}\left(1^{\prime}\right)$ respectively. It is worth to mention that one-dimensional ${ }^{1} \mathrm{H}-\mathrm{NMR}$ spectra along is not enough to assign the site of alkylation since chemical shifts of $\mathrm{N}(1)-\mathrm{CH}_{2}(5.81 \mathrm{ppm})$ and $\mathrm{O}(2)-\mathrm{CH}_{2}(5.76 \mathrm{ppm})$ are not distinguishable. However, one-dimensional ${ }^{13} \mathrm{C}$-NMR spectra provide very useful informations in which $\mathrm{C}\left(1^{\prime}\right)$ showed a upfield shift at $48.68 \mathrm{ppm}$ for $\mathrm{N}_{1}$-alkylated product 13a and a downfield shift at $67.48 \mathrm{ppm}$ for $\mathrm{O}_{2}$-alkylated product 13b. A view of a single molecule of 13a and $\mathbf{1 3} \mathbf{b}$ respectively are given in Figure 1.

Under the same alkylating conditions, quinolin-2(1H)-one substituted with an electron-donating group such as methoxy 3 , benzyloxy $\mathbf{4}$, or with an electron-withdrawing group such as chloro $\mathbf{5}$, at $\mathrm{C}(6)$-position gave a mixture of $\mathrm{N}_{1}$ - and $\mathrm{O}_{2}$ - alkylated products with the $\mathrm{N}_{1}$ - alkylation as the major product as shown in Scheme 1. Therefore, the electronic environment did not affect the type of alkylation. Accordingly, alkylation of C(7)-substituted quinolin$2(1 H)$-ones $\mathbf{6}, \mathbf{7}$, and $\mathbf{8}$ afforded $\mathrm{N}_{1}$-alkylated products 18a, 19a, and 20a as major products.

However, this type of alkylation can not be applied to C(8)-substituted quinolin-2(1H)-ones. Treatment of 8-methoxyquinolin-2(1H)-one (10) with 2 -bromoacetophenone and potassium carbonate in $\mathrm{DMF}$ gave $\mathrm{O}_{2}$-alkylated product, 8-methoxy-2-(2-oxo-2-phenylethoxy) quinoline (22b), as a sole product in a $75 \%$ yield. Accordingly, $\mathrm{O}_{2}$-alkylated products $\mathbf{2 1 \mathbf { b } ^ { 1 2 }}, \mathbf{2 3 \mathbf { b }}$, and 24b were obtained from the alkylation of their respective precursors 9, 11, and 12.

To confirm the application of this type of alkylation, further experiments were carried out by using chloroacetone as an alkylating agent as shown in Scheme 2. Treatment of $\mathrm{C}(6)$ and $\mathrm{C}(7)$ substituted quinolin-2(1H)-ones with chloroacetone and potassium carbonate in DMF gave a mixture of $\mathrm{N}_{1}$ - and $\mathrm{O}_{2}$ alkylated products with the former one as a major product. The typical peak of ${ }^{1} \mathrm{H}-\mathrm{NMR}$ spectra are not distinguishable, for example, $\mathrm{N}(1)-\mathrm{CH}_{2}(5.11 \mathrm{ppm})$ for 25a and $\mathrm{O}(2)-\mathrm{CH}_{2}(5.04 \mathrm{ppm})$ for $\mathbf{2 5} \mathbf{b}$. However, ${ }^{13} \mathrm{C}$-NMR spectra provide very useful informations in which $\mathrm{C}\left(1^{\prime}\right)$ showed a downfield shift at $70.07 \mathrm{ppm}$ for $\mathrm{O}_{2}$-alkylated product $\mathbf{2 5 b}$ and a upfield shift at $52.03 \mathrm{ppm}$ for $\mathrm{N}_{1}$-alkylated product 25a. Alkylation of $\mathrm{C}(8)$-substituted quinolin-2(1H)-ones under the same reaction conditions afforded $\mathrm{O}_{2}$-alkylated product as a sole product. Therefore, the site of alkylation could be controlled mostly by the steric effect 
in which less hindered $\mathrm{C}(6)$ and $\mathrm{C}(7)$ substituted quinolin-2(1H)-ones alkylated at $\mathrm{N}_{1}$ - and $\mathrm{O}_{2}$ - positions with the former one as a major product while $\mathrm{C}(8)$ substituted counterparts alkylated exclusively at less hindered $\mathrm{O}_{2}$-position.

Alkylating agents may also affect the regioselectivity in which the $\mathrm{N} / \mathrm{O}$ ratio for higher bulky 2-bromoacetophenone is less than that of less bulky chloroacetone due to higher steric hindrance of $\mathrm{N}$-alkylation than that of O-alkylation. For example, the N/O ratio is 2.7 (62/23) for 6-benzyloxy derivative (entry 4 , Scheme 1) using 2-bromoacetophenone as an alkylating agent while the N/O ratio is 7.1 (71/10) using chloroacetone (entry 4, Scheme 2) as an alkylating agent.

Scheme 1. Alkylation of quinolin-2(1H)-one with 2-bromoacetophenone.<smiles>[R]c1ccc2ccc(=O)[nH]c2c1</smiles>

\begin{tabular}{|c|c|c|c|}
\hline $\begin{array}{c}\text { Starting } \\
\text { material }\end{array}$ & $\mathrm{R}$ & Products & Yield (\%) \\
\hline $\mathbf{1}$ & $\mathrm{H}$ & $\mathbf{1 3 a} / \mathbf{1 3 b}$ & $66 / 7$ \\
\hline $\mathbf{2}$ & $6-\mathrm{OAc}$ & $\mathbf{1 4 a} / \mathbf{1 4 b}$ & $39 / 16$ \\
\hline $\mathbf{3}$ & $6-\mathrm{OMe}$ & $\mathbf{1 5 a} / \mathbf{1 5 b}$ & $80 / 7$ \\
\hline $\mathbf{4}$ & $6-\mathrm{OBn}$ & $\mathbf{1 6 a} / \mathbf{1 6 b}$ & $62 / 23$ \\
\hline $\mathbf{5}$ & $6-\mathrm{Cl}$ & $\mathbf{1 7 a} / \mathbf{1 7 b}$ & $64 / 28$ \\
\hline $\mathbf{6}$ & $7-\mathrm{OMe}$ & $\mathbf{1 8 a} / \mathbf{1 8 b}$ & $65 / 12$ \\
\hline $\mathbf{7}$ & $7-\mathrm{OBn}$ & $\mathbf{1 9 a} / \mathbf{1 9 b}$ & $79 / 13$ \\
\hline $\mathbf{8}$ & $7-\mathrm{Cl}$ & $\mathbf{2 0 a} / \mathbf{2 0 b}$ & $87 / 7$ \\
\hline $\mathbf{9}$ & $8-\mathrm{OAc}$ & $\mathbf{2 1 a} / \mathbf{2 1 b}$ & $0 / 74$ \\
\hline $\mathbf{1 0}$ & $8-\mathrm{OMe}$ & $\mathbf{2 2 a} / \mathbf{2 2 b}$ & $0 / 75$ \\
\hline $\mathbf{1 1}$ & $8-\mathrm{OBn}$ & $\mathbf{2 3 a} / \mathbf{2 3 b}$ & $0 / 91$ \\
\hline $\mathbf{1 2}$ & $8-\mathrm{Cl}$ & $\mathbf{2 4 a} / \mathbf{2 4 b}$ & $0 / 94$ \\
\hline
\end{tabular}

Scheme 2. Alkylation of quinolin-2(1H)-one with chloroacetone.<smiles>[Y]c1ccc2ccc(OCC(C)=O)nc2c1</smiles>

\begin{tabular}{|c|c|c|c|}
\hline $\begin{array}{c}\text { Starting } \\
\text { material }\end{array}$ & $\mathrm{R}$ & Products & Yield (\%) \\
\hline $\mathbf{1}$ & $\mathrm{H}$ & $\mathbf{2 5 a} / \mathbf{2 5 b}$ & $67 / 10$ \\
\hline $\mathbf{2}$ & $6-\mathrm{OAc}$ & $\mathbf{2 6 a} / \mathbf{2 6} \mathbf{b}$ & $75 / 6$ \\
\hline $\mathbf{3}$ & $6-\mathrm{OMe}$ & $\mathbf{2 7 a} / \mathbf{2 7} \mathbf{b}$ & $56 / 7$ \\
\hline $\mathbf{4}$ & $6-\mathrm{OBn}$ & $\mathbf{2 8 a} / \mathbf{2 8 b}$ & $71 / 10$ \\
\hline $\mathbf{5}$ & $6-\mathrm{Cl}$ & $\mathbf{2 9 a} / \mathbf{2 9 b}$ & $85 / 9$ \\
\hline $\mathbf{6}$ & $7-\mathrm{OMe}$ & $\mathbf{3 0 a} / \mathbf{3 0 b}$ & $84 / 8$ \\
\hline $\mathbf{7}$ & $7-\mathrm{OBn}$ & $\mathbf{3 1 a} / \mathbf{3 1 b}$ & $62 / 8$ \\
\hline $\mathbf{8}$ & $7-\mathrm{Cl}$ & $\mathbf{3 2 a} / \mathbf{3 2} \mathbf{b}$ & $76 / 8$ \\
\hline $\mathbf{9}$ & $8-\mathrm{OAc}$ & $\mathbf{3 3 a} / \mathbf{3 3 b}$ & $0 / 76$ \\
\hline $\mathbf{1 0}$ & $8-\mathrm{OMe}$ & $\mathbf{3 4 a} / \mathbf{3 4 b}$ & $0 / 67$ \\
\hline $\mathbf{1 1}$ & $8-\mathrm{OBn}$ & $\mathbf{3 5 a} / \mathbf{3 5 b}$ & $0 / 75$ \\
\hline $\mathbf{1 2}$ & $8-\mathrm{Cl}$ & $\mathbf{3 6 a} / \mathbf{3 6} \mathbf{b}$ & $0 / 89$ \\
\hline
\end{tabular}

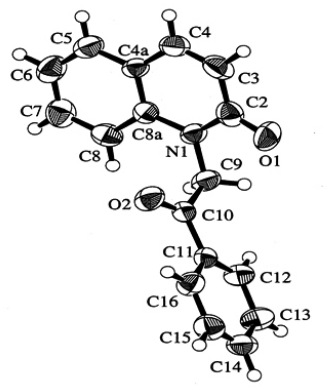

$13 \mathbf{a}$

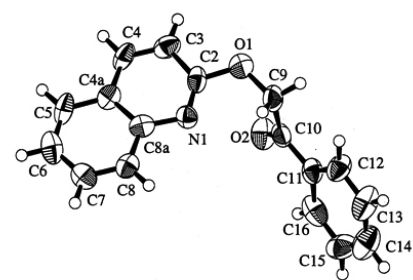

13b
Figure 1. X-Ray crystallographic structures of $13 \mathbf{a}$ and $\mathbf{1 3 b}$

\section{CONCLUSIONS}

In conclusion, we report herein the selective alkylation of certain substituted quinolin-2(1H)-one derivatives. Our results indicated that alkylation of relatively bulky 8-methoxy-, 8-benzyloxy-, and 8-chloro- quinolin-2(1H)ones under classical conditions (2-bromoacetophenone or chloroacetone, $\mathrm{DMF}, \mathrm{K}_{2} \mathrm{CO}_{3}$ ) gave exclusively $\mathrm{O}_{2}$-alkylated products. This selectivity can not be applied to $\mathrm{C}(6)$ and $\mathrm{C}(7)$ substituted quinolin-2(1H)-one counterparts in which alkylation occurred at both $\mathrm{N}_{1}$ - and $\mathrm{O}_{2}$ - positions with the former one as a major product. Therefore, alkylation of substituted quinolin-2(1H)-ones was controlled by the steric effect but not the electronic effect.

\section{EXPERIMENTAL}

TLC: Precoated $(0.2 \mathrm{~mm})$ silica gel $60-\mathrm{F}_{254}$ plates from EM Laboratories, Inc.; detection by UV light $(254 \mathrm{~nm})$. M.p.: Electrothermal IA9100 digital melting-point apparatus; uncorrected. ${ }^{1} \mathrm{H}$ and ${ }^{13} \mathrm{C}-\mathrm{NMR}$ spectra: VarianUnity-400 spectrometer at 400 and $100 \mathrm{MHz}$ or Varian-Gemini-200 spectrometer at 200 and $50 \mathrm{MHz}$, chemical shifts $d$ in ppm with $\mathrm{SiMe}_{4}$ as an internal standard (= 0 ppm), coupling constants $J$ in Hz. Elemental analyses were carried out on a Heraeus CHN-O-Rapid elemental analyzer, and results were within $\pm 0.4 \%$ of calculated values.

1-(2-Oxo-2-phenylethyl)quinolin-2(1H)-one (13a) and 2-(2-Oxo-2phenylethoxy)quinoline (13b)

Quinolin-2(1H)-one (1.45 g, $10 \mathrm{mmol}), \mathrm{K}_{2} \mathrm{CO}_{3}(1.38 \mathrm{~g}, 10 \mathrm{mmol})$, and dry DMF $(50 \mathrm{~mL})$ were stirred at $\mathrm{rt}$ for $30 \mathrm{~min}$. To this solution was added 2-bromoacetophenone $(1.99 \mathrm{~g}, 10 \mathrm{mmol})$ in dry DMF $(10 \mathrm{~mL})$ in one portion. The resulting mixture was continued to stir at $\mathrm{rt}$ for $24 \mathrm{~h}$ (TLC monitoring), and then poured into ice-water $(100 \mathrm{~mL})$. The mixture was extracted with $\mathrm{CH}_{2} \mathrm{Cl}_{2}(3 \times 75 \mathrm{~mL})$. The organic layer was combined, washed with $\mathrm{H}_{\mathrm{O}} \mathrm{O}$, dried $\left(\mathrm{Na}_{2} \mathrm{SO}_{4}\right)$, and then evaporated to give a brown solid which was purified by column chromatography on silica gel (AcOEt/Hexane 1:1). The proper fractions were combined and evaporated to furnish a residual solid which was crystallized from $\mathrm{CH}_{2} \mathrm{Cl}_{2} / \mathrm{Et}_{2} \mathrm{O}$ 1:10 to afford 13a $(1.74 \mathrm{~g}, 66 \%)$ and $\mathbf{1 3 b}(0.17$ $\mathrm{g}, 7 \%)$.

13a: $\mathrm{Mp} 164-165^{\circ} \mathrm{C} .{ }^{1} \mathrm{H}$ NMR (400 MHz, $\mathrm{CDCl}_{3}$ ): $\delta 5.81$ (s, $2 \mathrm{H}, \mathrm{H}-\mathrm{C}\left(1^{\prime}\right)$ ), $6.75(\mathrm{~d}, 1 \mathrm{H}, J=9.6 \mathrm{~Hz}, \mathrm{H}-\mathrm{C}(3)), 6.98(\mathrm{~d}, 1 \mathrm{H}, J=9.6 \mathrm{~Hz}, \mathrm{ArH}), 7.19-7.22(\mathrm{~m}$, 1H, ArH), 7.42-7.67 (m, 5H, ArH), 7.75 (d, $1 \mathrm{H}, J=9.6 \mathrm{~Hz}, \mathrm{H}-\mathrm{C}(4)), 8.08-8.10$ $(\mathrm{m}, 2 \mathrm{H}, \mathrm{ArH}) .{ }^{13} \mathrm{C}$ NMR $\left(100 \mathrm{MHz}, \mathrm{CDCl}_{3}\right): \delta 48.68\left(\mathrm{C}\left(1^{\prime}\right)\right), 114.02,120.78$, $121.17,122.26,128.10,128.89,129.02,130.68,133.95,134.87,139.55$, 139.86, $162.11(\mathrm{C}(2)), 192.37\left(\mathrm{C}\left(2^{\prime}\right)\right)$. Anal. Calcd for $\mathrm{C}_{17} \mathrm{H}_{13} \mathrm{NO}_{2}: \mathrm{C}, 77.55 ; \mathrm{H}$, 4.98; N, 5.32. Found: C, 77.52; H, 5.01; N, 5.35.

13b: $\mathrm{Mp} 115-116^{\circ} \mathrm{C} .{ }^{1} \mathrm{H}$ NMR $\left(200 \mathrm{MHz}, \mathrm{CDCl}_{3}\right): \delta 5.76\left(\mathrm{~s}, 2 \mathrm{H}, \mathrm{H}-\mathrm{C}\left(1^{\prime}\right)\right)$, $7.10(\mathrm{~d}, 1 \mathrm{H}, J=8.8 \mathrm{~Hz}, \mathrm{H}-\mathrm{C}(3)), 7.32-7.73(\mathrm{~m}, 7 \mathrm{H}, \mathrm{ArH}), 8.01-8.08(\mathrm{~m}, 3 \mathrm{H}$, $\mathrm{ArH}) .{ }^{13} \mathrm{C}$ NMR $\left(50 \mathrm{MHz}, \mathrm{CDCl}_{3}\right): \delta 67.48\left(\mathrm{C}\left(1^{\prime}\right)\right), 112.75,124.28,125.42$, $127.24,127.40,127.94,128.75,129.49,133.49,135.08,139.23,146.02$, $160.68(\mathrm{C}(2)), 194.49\left(\mathrm{C}\left(2^{\prime}\right)\right)$. Anal. Calcd for $\mathrm{C}_{17} \mathrm{H}_{13} \mathrm{NO}_{2}$ : C, 77.55; H, 4.98; N, 5.32. Found: C, 77.40; H, 5.00; N, 5.31.

6-Methoxy-1-(2-oxo-2-phenylethyl)quinolin-2(1H)-one (15a) and 6-Methoxy-2-(2-oxo-2-phenylethoxy)quinoline (15b)

Prepared from 6-methoxyquinolin-2 $(1 H)$-one and 2-bromoacetophenone by the same procedure as described for 13a and 13b. 15a: $80 \%$ yield. $\mathrm{Mp}$ 196-197 ${ }^{\circ} \mathrm{C} .{ }^{1} \mathrm{H}$ NMR (400 MHz, $\mathrm{CDCl}_{3}$ ): $\delta 3.83$ (s, 3H, MeO), 5.79 (s, $2 \mathrm{H}$, $\left.\mathrm{H}-\mathrm{C}\left(1^{\prime}\right)\right), 6.77(\mathrm{~d}, 1 \mathrm{H}, J=9.6 \mathrm{~Hz}, \mathrm{H}-\mathrm{C}(3)), 6.92(\mathrm{~d}, 1 \mathrm{H}, J=9.2 \mathrm{~Hz}, \mathrm{H}-\mathrm{C}(8))$, $7.02(\mathrm{~d}, 1 \mathrm{H}, J=2.8 \mathrm{~Hz}, \mathrm{H}-\mathrm{C}(5)), 7.06(\mathrm{dd}, 1 \mathrm{H}, J=9.2,2.8 \mathrm{~Hz}, \mathrm{H}-\mathrm{C}(7)), 7.51-$ 
7.54 (m, 2H, ArH), 7.62-7.66 (m, 1H, ArH), 7.69 (d, 1H, $J=9.2 \mathrm{~Hz}, \mathrm{H}-\mathrm{C}(4))$, 8.06-8.09 (m, 2H, ArH). ${ }^{13} \mathrm{C}$ NMR (100 MHz, $\left.\mathrm{CDCl}_{3}\right): \delta 48.84(\mathrm{C}(1 \phi)), 55.65$ $(\mathrm{MeO}), 110.77,115.36,119.33,121.50,121.62,128.10,128.89,133.96$, 134.00, 134.84, 139.39, 154.79, $161.72(\mathrm{C}(2)), 192.54(\mathrm{C}(2 \notin))$. Anal. Calcd for $\mathrm{C}_{18} \mathrm{H}_{15} \mathrm{NO}_{3}: \mathrm{C}, 73.71 ; \mathrm{H}, 5.15 ; \mathrm{N}, 4.78$. Found: $\mathrm{C}, 73.65 ; \mathrm{H}, 5.15 ; \mathrm{N}, 4.68$.

15b: $7 \%$ yield. Mp $94-95{ }^{\circ} \mathrm{C} .{ }^{1} \mathrm{H}$ NMR $\left(400 \mathrm{MHz}, \mathrm{CDCl}_{3}\right): \delta 3.88(\mathrm{~s}, 3 \mathrm{H}$, $\mathrm{MeO}), 5.74$ (s, 2H, H-C(1ф)), 7.04 (d, 1H, $J=2.4 \mathrm{~Hz}, \mathrm{H}-\mathrm{C}(5)), 7.08$ (d, $1 \mathrm{H}, J$ $=8.8 \mathrm{~Hz}, \mathrm{H}-\mathrm{C}(3)), 7.23(\mathrm{dd}, 1 \mathrm{H}, J=8.8,2.4 \mathrm{~Hz}, \mathrm{H}-\mathrm{C}(7)), 7.50-7.54(\mathrm{~m}, 2 \mathrm{H}$, $\mathrm{ArH}), 7.60-7.62(\mathrm{~m}, 1 \mathrm{H}, \mathrm{ArH}), 7.63(\mathrm{~d}, 1 \mathrm{H}, J=8.8 \mathrm{~Hz}, \mathrm{H}-\mathrm{C}(8)), 7.95(\mathrm{~d}, 1 \mathrm{H}$, $J=8.8 \mathrm{~Hz}, \mathrm{H}-\mathrm{C}(4)), 8.05-8.07$ (m, 2H, ArH). ${ }^{13} \mathrm{C}$ NMR (100 MHz, $\left.\mathrm{CDCl}_{3}\right): \delta$ $55.75(\mathrm{MeO}), 67.74(\mathrm{C}(1 \notin)), 106.33,113.10,121.46,126.23,128.19,128.70$ 129.01, 133.76, 135.32, 138.57, 141.55, 156.52, $159.63(\mathrm{C}(2)), 194.95(\mathrm{C}(2 \notin))$. Anal. Calcd for $\mathrm{C}_{18} \mathrm{H}_{15} \mathrm{NO}_{3}: \mathrm{C}, 73.71 ; \mathrm{H}, 5.15 ; \mathrm{N}, 4.78$. Found: $\mathrm{C}, 73.78 ; \mathrm{H}$, $5.29 ; \mathrm{N}, 4.60$

6-Benzyloxy-1-(2-oxo-2-phenylethyl)quinolin-2(1H)-one (16a) and 6-Benzyloxy-2-(2-oxo-2-phenylethoxy)quinoline (16b)

Prepared from 6-benzyloxyquinolin-2(1H)-one and 2-bromoacetophenone by the same procedure as described for 13a and 13b. 16a: $62 \%$ yield. $\mathrm{Mp}$ 177-178 ${ }^{\circ} \mathrm{C} .{ }^{1} \mathrm{H}$ NMR $\left(400 \mathrm{MHz}, \mathrm{CDCl}_{3}\right): \delta 5.09\left(\mathrm{~s}, 2 \mathrm{H}, \mathrm{CH}_{2} \mathrm{O}\right), 5.78(\mathrm{~s}, 2 \mathrm{H}$ $\mathrm{H}-\mathrm{C}(1 \notin)), 6.76(\mathrm{~d}, 1 \mathrm{H}, J=9.2 \mathrm{~Hz}, \mathrm{H}-\mathrm{C}(3)), 6.91(\mathrm{~d}, 1 \mathrm{H}, J=9.2 \mathrm{~Hz}, \mathrm{H}-\mathrm{C}(8))$, $7.10(\mathrm{~d}, 1 \mathrm{H}, J=2.8 \mathrm{~Hz}, \mathrm{H}-\mathrm{C}(5)), 7.13(\mathrm{dd}, 1 \mathrm{H}, J=8.8,2.8 \mathrm{~Hz}, \mathrm{H}-\mathrm{C}(7)), 7.31-$ $7.44(\mathrm{~m}, 5 \mathrm{H}, \mathrm{ArH}), 7.51-7.55(\mathrm{~m}, 2 \mathrm{H}, \mathrm{ArH}), 7.63-7.68$ (m, 2H, ArH), 8.07-8.09 $(\mathrm{m}, 1 \mathrm{H}, \mathrm{ArH}), 8.08(\mathrm{~d}, 1 \mathrm{H}, J=9.2 \mathrm{~Hz}, \mathrm{H}-\mathrm{C}(4)) \cdot{ }^{13} \mathrm{C} \mathrm{NMR}\left(100 \mathrm{MHz}, \mathrm{CDCl}_{3}\right): \delta$ $48.87(\mathrm{C}(1 \mathrm{c})), 70.55\left(\mathrm{CH}_{2} \mathrm{O}\right), 112.19,115.37,119.98,121.49,121.77,127.42$, $128.15,128.66,128.92,133.99,134.23,134.84,136.52,139.39,153.91$ $161.77(\mathrm{C}(2)), 192.55(\mathrm{C}(2 \notin))$. Anal. Calcd for $\mathrm{C}_{24} \mathrm{H}_{19} \mathrm{NO}_{3}$ : C, 78.03; H, 5.18; $\mathrm{N}, 3.79$. Found: $\mathrm{C}, 77.68 ; \mathrm{H}, 5.21 ; \mathrm{N}, 3.66$.

16b: $23 \%$ yield. Mp $145-146{ }^{\circ} \mathrm{C} .{ }^{1} \mathrm{H}$ NMR $\left(400 \mathrm{MHz}, \mathrm{CDCl}_{3}\right): \delta 5.14(\mathrm{~s}$, $\left.2 \mathrm{H}, \mathrm{CH}_{2} \mathrm{O}\right), 5.75(\mathrm{~s}, 2 \mathrm{H}, \mathrm{H}-\mathrm{C}(1 \phi)), 7.09(\mathrm{~d}, 1 \mathrm{H}, J=8.8 \mathrm{~Hz}, \mathrm{H}-\mathrm{C}(3)), 7.13(\mathrm{~d}$, $1 \mathrm{H}, J=2.8 \mathrm{~Hz}, \mathrm{H}-\mathrm{C}(5)), 7.32(\mathrm{dd}, 1 \mathrm{H}, J=9.2,2.8 \mathrm{~Hz}, \mathrm{H}-\mathrm{C}(7)), 7.34-7.37(\mathrm{~m}$ $1 \mathrm{H}, \mathrm{ArH}), 7.38-7.43(\mathrm{~m}, 2 \mathrm{H}, \mathrm{ArH}), 7.46-7.49(\mathrm{~m}, 2 \mathrm{H}, \mathrm{ArH}), 7.51-7.54(\mathrm{~m}, 2 \mathrm{H}$ ArH), 7.61-7.63 (m, 1H, ArH), $7.64(\mathrm{~d}, 1 \mathrm{H}, J=9.2 \mathrm{~Hz}, \mathrm{H}-\mathrm{C}(8)), 7.95(\mathrm{~d}, 1 \mathrm{H}$, $J=8.8 \mathrm{~Hz}, \mathrm{H}-\mathrm{C}(4)), 8.05-8.08(\mathrm{~m}, 2 \mathrm{H}, \mathrm{ArH}) .{ }^{13} \mathrm{C} \mathrm{NMR}\left(100 \mathrm{MHz}, \mathrm{CDCl}_{3}\right): \delta$ $67.83(\mathrm{C}(1 \varnothing)), 70.54\left(\mathrm{CH}_{2} \mathrm{O}\right), 107.82,113.16,121.91,126.20,127.78,128.20$ $128.32,128.74,128.88,129.02,133.77,135.31,136.95,138.66,141.61$, 155.68, $159.71(\mathrm{C}(2))$, $194.89(\mathrm{C}(2 \phi))$. Anal. Calcd for $\mathrm{C}_{24} \mathrm{H}_{19} \mathrm{NO}_{3}$ : C, 78.03; H, 5.18; N, 3.79. Found: C, 78.00; H, 5.20; N, 3.78

6-Chloro-1-(2-oxo-2-phenylethyl)quinolin-2(1H)-one (17a) and 6-Chloro-2-(2-oxo-2-phenylethoxy)quinoline (17b)

Prepared from 6-chloroquinolin-2(1H)-one and 2-bromoacetophenone by the same procedure as described for 13a and 13b. 17a: $64 \%$ yield. $\mathrm{Mp} 213$ $214{ }^{\circ} \mathrm{C} .{ }^{1} \mathrm{H}$ NMR $\left(200 \mathrm{MHz}, \mathrm{CDCl}_{3}\right): \delta 5.78(\mathrm{~s}, 2 \mathrm{H}, \mathrm{H}-\mathrm{C}(1 c)), 6.78(\mathrm{~d}, 1 \mathrm{H}, J$ $=9.5 \mathrm{~Hz}, \mathrm{H}-\mathrm{C}(3)), 6.91(\mathrm{~d}, 1 \mathrm{H}, J=9.0 \mathrm{~Hz}, \mathrm{H}-\mathrm{C}(8)), 7.38(\mathrm{dd}, 1 \mathrm{H}, J=9.0,2.4$ $\mathrm{Hz}, \mathrm{H}-\mathrm{C}(7)), 7.50-7.57(\mathrm{~m}, 2 \mathrm{H}, \mathrm{ArH}), 7.56(\mathrm{~d}, 1 \mathrm{H}, J=2.4 \mathrm{~Hz}, \mathrm{H}-\mathrm{C}(5)), 7.63-$ $7.67(\mathrm{~m}, 1 \mathrm{H}, \mathrm{ArH}), 7.87(\mathrm{~d}, 1 \mathrm{H}, J=9.5 \mathrm{~Hz}, \mathrm{H}-\mathrm{C}(4)), 8.05-8.10(\mathrm{~m}, 2 \mathrm{H}, \mathrm{ArH})$. ${ }^{13} \mathrm{C}$ NMR (50 MHz, $\left.\mathrm{CDCl}_{3}\right): \delta 48.82(\mathrm{C}(1 \notin)), 115.57,121,79,122.54,127.78$, $128.09,128.16,129.00,130.65,134.19,134.69,138.16,138.72,161.86(\mathrm{C}(2))$, $192.07(\mathrm{C}(2 \phi))$. Anal. Calcd for $\mathrm{C}_{17} \mathrm{H}_{12} \mathrm{NO}_{2} \mathrm{Cl}: \mathrm{C}, 68.58 ; \mathrm{H}, 4.06 ; \mathrm{N}, 4.70$. Found: C, $68.47 ; \mathrm{H}, 4.07 ; \mathrm{N}, 4.77$

17b: $28 \%$ yield. Mp $123-124{ }^{\circ} \mathrm{C} .{ }^{1} \mathrm{H}$ NMR $\left(400 \mathrm{MHz}, \mathrm{CDCl}_{3}\right): \delta 5.76$ (s, $2 \mathrm{H}, \mathrm{H}-\mathrm{C}(1 \mathrm{c})), 7.13(\mathrm{~d}, 1 \mathrm{H}, J=8.8 \mathrm{~Hz}, \mathrm{H}-\mathrm{C}(3)), 7.49$ (dd, $1 \mathrm{H}, J=8.8$ $2.4 \mathrm{~Hz}, \mathrm{H}-\mathrm{C}(7)), 7.51-7.55$ (m, 2H, ArH), 7.61-7.66 (m, 2H, ArH), 7.69 (d, $1 \mathrm{H}, J=2.4 \mathrm{~Hz}, \mathrm{H}-\mathrm{C}(5)), 7.96(\mathrm{~d}, 1 \mathrm{H}, J=8.8 \mathrm{~Hz}, \mathrm{H}-\mathrm{C}(8)), 8.05(\mathrm{~d}, 1 \mathrm{H}, J$ $=8.8 \mathrm{~Hz}, \mathrm{H}-\mathrm{C}(4)), 8.04-8.06(\mathrm{~m}, 1 \mathrm{H}, \mathrm{ArH}) .{ }^{13} \mathrm{C} \mathrm{NMR}\left(100 \mathrm{MHz}, \mathrm{CDCl}_{3}\right): \delta$ $67.80(\mathrm{C}(1 \notin)), 114.06,126.27,126.54,128.16,129.01,129.07,129.94,130.42$, $133.89,135.15,138.56,144.69,160.14(\mathrm{C}(2)), 194.45(\mathrm{C}(2)))$. Anal. Calcd for $\mathrm{C}_{17} \mathrm{H}_{12} \mathrm{NO}_{2} \mathrm{Cl}: \mathrm{C}, 68.58 ; \mathrm{H}, 4.06 ; \mathrm{N}, 4.70$. Found: $\mathrm{C}, 68.45 ; \mathrm{H}, 4.08 ; \mathrm{N}, 4.73$.

7-Methoxy-1-(2-oxo-2-phenylethyl)quinolin-2(1H)-one (18a) and 7-Methoxy-2-(2-oxo-2-phenylethoxy)quinoline (18b)

Prepared from 7-methoxyquinolin-2(1H)-one and 2-bromoacetophenone by the same procedure as described for 13a and 13b. 18a: $65 \%$ yield. $\mathrm{Mp}$ $171-172{ }^{\circ} \mathrm{C} .{ }^{1} \mathrm{H}$ NMR $\left(400 \mathrm{MHz}, \mathrm{CDCl}_{3}\right): \delta 3.78(\mathrm{~s}, 3 \mathrm{H}, \mathrm{MeO}), 5.76(\mathrm{~s}, 2 \mathrm{H}$, H-C $(1 \notin)), 6.44(\mathrm{~d}, 1 \mathrm{H}, J=2.4 \mathrm{~Hz}, \mathrm{H}-\mathrm{C}(8)), 6.60(\mathrm{~d}, 1 \mathrm{H}, J=9.2 \mathrm{~Hz}, \mathrm{H}-\mathrm{C}(3))$, $6.80(\mathrm{dd}, 1 \mathrm{H}, J=8.8,2.4 \mathrm{~Hz}, \mathrm{H}-\mathrm{C}(6)), 7.49(\mathrm{~d}, 1 \mathrm{H}, J=8.8 \mathrm{~Hz}, \mathrm{H}-\mathrm{C}(5)), 7.51$ 7.55 (m, 2H, ArH), 7.63-7.65 (m, 1H, ArH), 7.68 (d, 1H, $J=9.6 \mathrm{~Hz}, \mathrm{H}-\mathrm{C}(4))$, $8.07-8.10(\mathrm{~m}, 2 \mathrm{H}, \mathrm{ArH}) .{ }^{13} \mathrm{C}$ NMR $\left(100 \mathrm{MHz}, \mathrm{CDCl}_{3}\right): \delta 49.14(\mathrm{C}(1 \notin))$, $55.74(\mathrm{MeO}), 99.42,109.60,115.34,118.24,128.38,129.18,130.69,134.25$, 135.14, 139.98, 141.45, 162.08, 162.77 (C(2)), 192.95 (C(2屯)). Anal. Calcd for $\mathrm{C}_{4} \mathrm{HO}$ : C, 73.71; H, 5.15; N, 4.78. Found: $\mathrm{C}, 73.70 ; \mathrm{H}, 5.17 ; \mathrm{N}, 4.78$.

18b: $12 \%$ yield. Mp 137-138 ${ }^{\circ} \mathrm{C} .{ }^{1} \mathrm{H}$ NMR (400 MHz, $\left.\mathrm{CDCl}_{3}\right): \delta 3.87$ (s, $3 \mathrm{H}, \mathrm{MeO}), 5.77(\mathrm{~s}, 2 \mathrm{H}, \mathrm{H}-\mathrm{C}(1 \mathrm{c})), 6.96(\mathrm{~d}, 1 \mathrm{H}, J=8.8 \mathrm{~Hz}, \mathrm{H}-\mathrm{C}(3)), 7.01(\mathrm{dd}$, $1 \mathrm{H}, J=8.8,2.4 \mathrm{~Hz}, \mathrm{H}-\mathrm{C}(6)), 7.07$ (d, $1 \mathrm{H}, J=2.4 \mathrm{~Hz}, \mathrm{H}-\mathrm{C}(8)), 7.50-7.55$ (m, $2 \mathrm{H}, \mathrm{ArH}), 7.59(\mathrm{~d}, 1 \mathrm{H}, J=9.2 \mathrm{~Hz}, \mathrm{H}-\mathrm{C}(5)), 7.61-7.65(\mathrm{~m}, 1 \mathrm{H}, \mathrm{ArH}), 7.96(\mathrm{~d}$,
$1 \mathrm{H}, J=8.8 \mathrm{~Hz}, \mathrm{H}-\mathrm{C}(4)), 8.06-8.09$ (m, $2 \mathrm{H}, \mathrm{ArH}) .{ }^{13} \mathrm{C} \mathrm{NMR}\left(100 \mathrm{MHz}, \mathrm{CDCl}_{3}\right)$ : $\delta 55.64(\mathrm{MeO}), 67.78(\mathrm{C}(1 \notin)), 106.76,110.24,116.69,120.47,128.22,128.66$, $129.00,133.77,135.27,139.16,148.05,161.20,161.51(C(2)), 194.75(C(2 \phi))$. Anal. Calcd for $\mathrm{C}_{18} \mathrm{H}_{15} \mathrm{NO}_{3}$ : C, 73.71; H, 5.15; N, 4.78. Found: C, 73.47; H, $5.24 ; \mathrm{N}, 4.39$.

7-Benzyloxy-1-(2-oxo-2-phenylethyl)quinolin-2(1H)-one (19a) and 7-Benzyloxy-2-(2-oxo-2-phenylethoxy)quinoline (19b)

Prepared from 7-benzyloxyquinolin-2(1H)-one and 2-bromoacetophenone by the same procedure as described for 13a and 13b. 19a: $79 \%$ yield. Mp $185-186{ }^{\circ} \mathrm{C} .{ }^{1} \mathrm{H}$ NMR $\left(400 \mathrm{MHz}, \mathrm{CDCl}_{3}\right): \delta 4.99\left(\mathrm{~s}, 2 \mathrm{H}, \mathrm{CH}_{2} \mathrm{O}\right), 5.66(\mathrm{~s}, 2 \mathrm{H}$, $\mathrm{H}-\mathrm{C}(1 \notin)), 6.45(\mathrm{~d}, 1 \mathrm{H}, J=2.4 \mathrm{~Hz}, \mathrm{H}-\mathrm{C}(8)), 6.57(\mathrm{~d}, 1 \mathrm{H}, J=9.6 \mathrm{~Hz}, \mathrm{H}-\mathrm{C}(3))$, $6.83(\mathrm{dd}, 1 \mathrm{H}, J=8.8,2.4 \mathrm{~Hz}, \mathrm{H}-\mathrm{C}(6)), 7.25-7.28(\mathrm{~m}, 5 \mathrm{H}, \mathrm{ArH}), 7.45(\mathrm{~d}, 1 \mathrm{H}, J=$ $8.8 \mathrm{~Hz}, \mathrm{H}-\mathrm{C}(5)), 7.48-7.51(\mathrm{~m}, 2 \mathrm{H}, \mathrm{ArH}), 7.60-7.65(\mathrm{~m}, 2 \mathrm{H}, \mathrm{ArH}), 8.01(\mathrm{~d}, 1 \mathrm{H}$, $J=9.6 \mathrm{~Hz}, \mathrm{H}-\mathrm{C}(4)), 8.02-8.03(\mathrm{~m}, 1 \mathrm{H}, \mathrm{ArH}) .{ }^{13} \mathrm{C}$ NMR (100 MHz, $\left.\mathrm{CDCl}_{3}\right): \delta$ $49.19(\mathrm{C}(1 \mathrm{~d})), 70.65\left(\mathrm{CH}_{2} \mathrm{O}\right), 100.38,110.55,115.48,118.30,127.68,128.41$, $128.50,128.94,129.17,130.67,134.29,135.07,136.28,139.98,141.38$, 161.21, $162.77(\mathrm{C}(2)), 192.65(\mathrm{C}(2 \notin))$. Anal. Calcd for $\mathrm{C}_{24} \mathrm{H}_{19} \mathrm{NO}_{3}$ : C, 78.03; H, 5.18; N, 3.79. Found: C, 78.00; H, 5.21; N, 3.79.

19b: $13 \%$ yield. Mp $146-147^{\circ} \mathrm{C} .{ }^{1} \mathrm{H}$ NMR $\left(400 \mathrm{MHz}, \mathrm{CDCl}_{3}\right): \delta 5.12(\mathrm{~s}$, $2 \mathrm{H}, \mathrm{CH}_{2} \mathrm{O}$ ), 5.77 (s, 2H, H-C(1)), 6.97 (d, $\left.1 \mathrm{H}, J=9.6 \mathrm{~Hz}, \mathrm{H}-\mathrm{C}(3)\right), 7.10$ (dd, $1 \mathrm{H}, J=8.8,2.4 \mathrm{~Hz}, \mathrm{H}-\mathrm{C}(6)), 7.17$ (d, $1 \mathrm{H}, J=2.4 \mathrm{~Hz}, \mathrm{H}-\mathrm{C}(8)), 7.32-7.42(\mathrm{~m}$, $3 \mathrm{H}, \mathrm{ArH}), 7.44-7.47(\mathrm{~m}, 2 \mathrm{H}, \mathrm{ArH}), 7.51-7.55(\mathrm{~m}, 2 \mathrm{H}, \mathrm{ArH}), 7.61-7.66(\mathrm{~m}$, $2 \mathrm{H}, \mathrm{ArH}), 7.97(\mathrm{~d}, 1 \mathrm{H}, J=8.8 \mathrm{~Hz}, \mathrm{H}-\mathrm{C}(5)), 8.06-8.09(\mathrm{~m}, 1 \mathrm{H}, \mathrm{ArH}), 8.07$ (d, $1 \mathrm{H}, J=9.6 \mathrm{~Hz}, \mathrm{H}-\mathrm{C}(4)) .{ }^{13} \mathrm{C}$ NMR $\left(100 \mathrm{MHz}, \mathrm{CDCl}_{3}\right): \delta 67.83(\mathrm{C}(1 \mathrm{c})), 70.29$ $\left(\mathrm{CH}_{2} \mathrm{O}\right), 107.91,110.38,117.04,120.62,127.88,128.23,128.33,128.75$, $128.86,128.93,129.01,133.78,135.30,136.80,139.19,160.35,161.51(\mathrm{C}(2))$, 194.75 (C(2ø)). Anal. Calcd for $\mathrm{C}_{24} \mathrm{H}_{19} \mathrm{NO}_{3}: \mathrm{C}, 78.03 ; \mathrm{H}, 5.18 ; \mathrm{N}, 3.79$. Found: C, 78.40; H, 5.26; N, 3.71

7-Chloro-1-(2-oxo-2-phenylethyl)quinolin-2(1H)-one

(20a) and 7-Chloro-2-(2-0xo-2-phenylethoxy)quinoline (20b)

Prepared from 7-chloroquinolin-2(1H)-one and 2-bromoacetophenone by the same procedure as described for 13a and 13b. 20a: $87 \%$ yield. Mp 180-181 ${ }^{\circ} \mathrm{C} .{ }^{1} \mathrm{H}$ NMR $(400 \mathrm{MHz}, \mathrm{CDCl})$ ) $\delta 5.76(\mathrm{~s}, 2 \mathrm{H}, \mathrm{H}-\mathrm{C}(1 \notin)), 6.75(\mathrm{~d}, 1 \mathrm{H}, J=9.2$ $\mathrm{Hz}, \mathrm{H}-\mathrm{C}(3)), 6.97$ (d, $1 \mathrm{H}, J=2.0 \mathrm{~Hz}, \mathrm{H}-\mathrm{C}(8)), 7.19$ (dd, $1 \mathrm{H}, J=8.4,2.0 \mathrm{~Hz}$, $\mathrm{H}-\mathrm{C}(6)), 7.52(\mathrm{~d}, 1 \mathrm{H}, J=8.4 \mathrm{~Hz}, \mathrm{H}-\mathrm{C}(5)), 7.55-7.59(\mathrm{~m}, 2 \mathrm{H}, \mathrm{ArH}), 7.66-7.71$ $(\mathrm{m}, 1 \mathrm{H}, \mathrm{ArH}), 7.72(\mathrm{~d}, 1 \mathrm{H}, J=9.2 \mathrm{~Hz}, \mathrm{H}-\mathrm{C}(4)), 8.09-8.12(\mathrm{~m}, 2 \mathrm{H}, \mathrm{ArH}) \cdot{ }^{13} \mathrm{C}$ NMR $\left(100 \mathrm{MHz}, \mathrm{CDCl}_{3}\right): \delta 49.05$ (C(1ф)), 114.37, 119.51, 121.49, 123.10, $128.46,129.27,130.34,134.48,134.87,137.21,139.57,140.71,162.15(\mathrm{C}(2))$, $192.04(\mathrm{C}(2 \phi))$. Anal. Calcd for $\mathrm{C}_{17} \mathrm{H}_{12} \mathrm{NO}_{2} \mathrm{Cl} \cdot 0.2 \mathrm{H}_{2} \mathrm{O}: \mathrm{C}, 67.76 ; \mathrm{H}, 4.14 ; \mathrm{N}$, 4.65. Found: C, 67.92; H, 4.02; N, 4.66 .

20b: $7 \%$ yield. Mp $106-107^{\circ} \mathrm{C} .{ }^{1} \mathrm{H}$ NMR $\left(400 \mathrm{MHz}, \mathrm{CDCl}_{3}\right): \delta 5.76(\mathrm{~s}$, $2 \mathrm{H}, \mathrm{H}-\mathrm{C}(1 \notin)), 7.10(\mathrm{~d}, 1 \mathrm{H}, J=8.8 \mathrm{~Hz}, \mathrm{H}-\mathrm{C}(3)), 7.32(\mathrm{dd}, 1 \mathrm{H}, J=8.4,2.0 \mathrm{~Hz}$, $\mathrm{H}-\mathrm{C}(6)), 7.51-7.55(\mathrm{~m}, 2 \mathrm{H}, \mathrm{ArH}), 7.62-7.66(\mathrm{~m}, 1 \mathrm{H}, \mathrm{ArH}), 7.64(\mathrm{~d}, 1 \mathrm{H}, J=8.4$ $\mathrm{Hz}, \mathrm{H}-\mathrm{C}(5)), 7.70(\mathrm{~d}, 1 \mathrm{H}, J=2.4 \mathrm{~Hz}, \mathrm{H}-\mathrm{C}(8)), 8.01(\mathrm{~d}, 1 \mathrm{H}, J=8.8 \mathrm{~Hz}, \mathrm{H}-\mathrm{C}(4))$, 8.03-8.06 (m, 2H, ArH). ${ }^{13} \mathrm{C}$ NMR (100 MHz, $\left.\mathrm{CDCl}_{3}\right): \delta 67.94(\mathrm{C}(1 \notin)), 113.26$, $123.99,125.46,126.65,128.16,128.79,129.10,133.94,135.07,135.65$, 139.29, 146.75, $161.66(\mathrm{C}(2)), 194.31(\mathrm{C}(2 \phi))$. Anal. Calcd for $\mathrm{C}_{17} \mathrm{H}_{12} \mathrm{NO}_{2} \mathrm{Cl}$ : C, 68.58; H, 4.06; N, 4.70. Found: C, 68.22; H, 4.05; N, 4.64

8-Methoxy-2-(2-oxo-2-phenylethoxy)quinoline (22b)

Prepared from 8-methoxyquinolin-2(1H)-one and 2-bromoacetophenone by the same procedure as described for 13a and 13b. $75 \%$ yield. Mp 161-162 ${ }^{\circ} \mathrm{C} .{ }^{1} \mathrm{H}$ NMR (400 MHz, $\mathrm{CDCl}_{3}$ ): $\delta 3.88$ (s, 3H, MeO), 5.80 (s, 2H, H-C $(1 \phi)$ ), 6.99-7.01 (m, 1H, ArH), $7.14(\mathrm{~d}, 1 \mathrm{H}, J=8.8 \mathrm{~Hz}, \mathrm{H}-\mathrm{C}(3)), 7.27-7.34(\mathrm{~m}, 2 \mathrm{H}$, ArH), 7.49-7.54 (m, 2H, ArH), 7.59-7.63 (m, 1H, ArH), $8.03(\mathrm{~d}, 1 \mathrm{H}, J=8.8$ $\mathrm{Hz}, \mathrm{H}-\mathrm{C}(4)), 8.07-8.10(\mathrm{~m}, 2 \mathrm{H}, \mathrm{ArH}) .{ }^{13} \mathrm{C}$ NMR $\left(100 \mathrm{MHz}, \mathrm{CDCl}_{3}\right): \delta 56.17$ (MeO), $67.53(\mathrm{C}(1 \notin)), 109.54,113.16,119.66,124.34,126.55,128.13,128.65$, 133.37, 135.46, 137.48, 139.42, 154.14, $160.16(\mathrm{C}(2)), 194.94$ (C(2ø)). Anal. Calcd for $\mathrm{C}_{18} \mathrm{H}_{15} \mathrm{NO}_{3}: \mathrm{C}, 73.71 ; \mathrm{H}, 5.15 ; \mathrm{N}, 4.78$. Found: $\mathrm{C}, 73.64 ; \mathrm{H}, 5.19 ; \mathrm{N}$, 4.80 .

\section{8-Benzyloxy-2-(2-oxo-2-phenylethoxy)quinoline (23b)}

Prepared from 8-benzyloxyquinolin-2(1H)-one and 2-bromoacetophenone by the same procedure as described for 13a and 13b. $91 \%$ yield. Mp 132-133 ${ }^{\circ} \mathrm{C} .{ }^{1} \mathrm{H}$ NMR $\left(400 \mathrm{MHz}, \mathrm{CDCl}_{3}\right): \delta 5.16\left(\mathrm{~s}, 2 \mathrm{H}, \mathrm{CH}_{2} \mathrm{O}\right), 5.86(\mathrm{~s}, 2 \mathrm{H}, \mathrm{H}-\mathrm{C}(1 \phi))$, $7.07(\mathrm{~d}, 1 \mathrm{H}, J=8.8 \mathrm{~Hz}, \mathrm{H}-\mathrm{C}(3)), 7.15-7.28(\mathrm{~m}, 5 \mathrm{H}, \mathrm{ArH}), 7.33-7.37(\mathrm{~m}$, $3 \mathrm{H}, \mathrm{ArH}), 7.44-7.48(\mathrm{~m}, 2 \mathrm{H}, \mathrm{ArH}), 7.59-7.62(\mathrm{~m}, 1 \mathrm{H}, \mathrm{ArH}), 8.02-8.06(\mathrm{~m}$, $2 \mathrm{H}, \mathrm{ArH}), 8.05(\mathrm{~d}, 1 \mathrm{H}, J=8.8 \mathrm{~Hz}, \mathrm{H}-\mathrm{C}(4)) .{ }^{13} \mathrm{C} \mathrm{NMR}\left(100 \mathrm{MHz}, \mathrm{CDCl}_{3}\right): \delta$ $67.81(\mathrm{C}(1 \notin)), 71.38\left(\mathrm{CH}_{2} \mathrm{O}\right), 112.83,113.26,120.62,124.48,126.94,127.16$, $127.72,128.15,128.53,128.71,129.02,133.71,135.27,137.61,139.65$, 153.42, $160.31(\mathrm{C}(2)), 194.62(\mathrm{C}(2 \notin))$. Anal. Calcd for $\mathrm{C}_{24} \mathrm{H}_{19} \mathrm{NO}_{3}$ : C, 78.03; H, 5.18; N, 3.79. Found: C, 78.01; H, 5.17; N, 3.61.

\section{8-Chloro-2-(2-oxo-2-phenylethoxy)quinoline (24b)}

Prepared from 8-chloroquinolin-2(1H)-one and 2-bromoacetophenone by the same procedure as described for 13a and 13b. $94 \%$ yield. Mp $183-184{ }^{\circ} \mathrm{C}$. 
${ }^{1} \mathrm{H}$ NMR $\left(400 \mathrm{MHz}, \mathrm{CDCl}_{3}\right): \delta 5.80(\mathrm{~s}, 2 \mathrm{H}, \mathrm{H}-\mathrm{C}(1 \not)), 7.16(\mathrm{~d}, 1 \mathrm{H}, J=8.8 \mathrm{~Hz}$, $\mathrm{H}-\mathrm{C}(3)), 7.24-7.28(\mathrm{~m}, 1 \mathrm{H}, \mathrm{ArH}), 7.49-7.53(\mathrm{~m}, 2 \mathrm{H}, \mathrm{ArH}), 7.59-7.67(\mathrm{~m}, 3 \mathrm{H}$, $\mathrm{ArH}), 8.04-8.07$ (m, 2H, ArH), 8.05 (d, $1 \mathrm{H}, J=8.8 \mathrm{~Hz}, \mathrm{H}-\mathrm{C}(4)) .{ }^{13} \mathrm{C}$ NMR $\left(100 \mathrm{MHz}, \mathrm{CDCl}_{2}\right): \delta 67.73(\mathrm{C}(1 \notin)), 113.54,124.25,126.33,126.58,127.99$, 128.74, 129.74, 131.44, 133.50, 135.28, 139.69, 142.38, 161.01 (C(2)), 194.45 $(\mathrm{C}(2 \notin))$. Anal. Calcd for $\mathrm{C}_{17} \mathrm{H}_{12} \mathrm{NO}_{2} \mathrm{Cl}$ : C, 68.58; H, 4.06; N, 4.70. Found: $\mathrm{C}$, $68.42 ; \mathrm{H}, 4.11 ; \mathrm{N}, 4.81$

1-(2-Oxopropyl)quinolin-2(1H)-one (25a) and 2-(2-Oxopropoxy) quinoline (25b)

Prepared from quinolin-2(1H)-one and chloroacetone by the same procedure as described for 13a and 13b. 25a: $67 \%$ yield. Mp $108-109{ }^{\circ} \mathrm{C} .{ }^{1} \mathrm{H}$ NMR (400 MHz, $\left.\mathrm{CDCl}_{3}\right): \delta 2.24(\mathrm{~s}, 3 \mathrm{H}, \mathrm{Me}), 5.11(\mathrm{~s}, 2 \mathrm{H}, \mathrm{H}-\mathrm{C}(1 \varnothing)), 6.73(\mathrm{~d}$, $1 \mathrm{H}, J=9.6 \mathrm{~Hz}, \mathrm{H}-\mathrm{C}(3)), 7.00-7.24(\mathrm{~m}, 2 \mathrm{H}, \mathrm{ArH}), 7.51-7.58(\mathrm{~m}, 2 \mathrm{H}, \mathrm{ArH})$, $7.74(\mathrm{~d}, 1 \mathrm{H}, J=9.6 \mathrm{~Hz}, \mathrm{H}-\mathrm{C}(4)) .{ }^{13} \mathrm{C}$ NMR $\left(100 \mathrm{MHz}, \mathrm{CDCl}_{3}\right): \delta 27.06(\mathrm{Me})$, $52.03(\mathrm{C}(1 \varnothing)), 113.69,120.69,121.20,122.51,129.12,130.90,139.30,139.95$, $161.89(\mathrm{C}(2))$, $202.49(\mathrm{C}(2 \phi))$. Anal. Calcd for $\mathrm{C}_{12} \mathrm{H}_{11} \mathrm{NO}_{2}: \mathrm{C}, 71.63 ; \mathrm{H}, 5.51$; $\mathrm{N}, 6.96$. Found: C, 71.66; H, 5.51; N, 6.94 .

25b: $10 \%$ yield. Oily. ${ }^{1} \mathrm{H}$ NMR $\left(200 \mathrm{MHz}, \mathrm{CDCl}_{3}\right): \delta 2.27$ (s, 3H, Me), $5.04(\mathrm{~s}, 2 \mathrm{H}, \mathrm{H}-\mathrm{C}(1 \mathrm{c})), 7.03(\mathrm{~d}, 1 \mathrm{H}, J=8.8 \mathrm{~Hz}, \mathrm{H}-\mathrm{C}(3)), 7.39-7.61(\mathrm{~m}, 2 \mathrm{H}$, $\mathrm{ArH}), 7.71-7.79(\mathrm{~m}, 2 \mathrm{H}, \mathrm{ArH}), 8.04(\mathrm{~d}, 1 \mathrm{H}, J=9.0 \mathrm{~Hz}, \mathrm{H}-\mathrm{C}(4)) .{ }^{13} \mathrm{C}$ NMR $(50$ $\left.\mathrm{MHz}, \mathrm{CDCl}_{3}\right): \delta 26.45(\mathrm{Me}), 70.07(\mathrm{C}(1 \notin)), 112.54,124.44,125.40,127.29$, 127.42, 129.64, 139.35, 146.00, 160.61 (C(2)), $204.89(\mathrm{C}(2 \phi))$. Anal. Calcd for $\mathrm{C}_{12} \mathrm{H}_{11} \mathrm{NO}_{2}: \mathrm{C}, 71.63 ; \mathrm{H}, 5.51 ; \mathrm{N}, 6.96$. Found: $\mathrm{C}, 71.33 ; \mathrm{H}, 5.71 ; \mathrm{N}, 6.83$.

6-Methoxy-1-(2-oxopropyl)quinolin-2(1H)-one (27a) and 6-Methoxy2-(2-oxopropoxy)quinoline (27b)

Prepared from 6-methoxyquinolin-2(1H)-one and chloroacetone by the same procedure as described for 13a and 13b. 27a: $56 \%$ yield. Mp $171-172{ }^{\circ} \mathrm{C}$. ${ }^{1} \mathrm{H} N M R\left(400 \mathrm{NHz}, \mathrm{CDCl}_{3}\right): \delta 2.22(3 \mathrm{H}, \mathrm{s}, \mathrm{Me}), 3.85(\mathrm{~s}, 3 \mathrm{H}, \mathrm{MeO}), 5.09(\mathrm{~s}, 2 \mathrm{H}$, $\mathrm{H}-\mathrm{C}(1 c)), 6.74(\mathrm{~d}, 1 \mathrm{H}, J=9.6 \mathrm{~Hz}, \mathrm{H}-\mathrm{C}(3)), 6.94(\mathrm{~d}, 1 \mathrm{H}, J=9.2 \mathrm{~Hz}, \mathrm{H}-\mathrm{C}(8))$, 7.03 (d, $1 \mathrm{H}, J=2.8 \mathrm{~Hz}, \mathrm{H}-\mathrm{C}(5)), 7.13$ (dd, $1 \mathrm{H}, J=9.2,2.8 \mathrm{~Hz}, \mathrm{H}-\mathrm{C}(7)), 7.68$ $(\mathrm{d}, 1 \mathrm{H}, J=9.6 \mathrm{~Hz}, \mathrm{H}-\mathrm{C}(4)) .{ }^{13} \mathrm{C}$ NMR $\left(100 \mathrm{MHz}, \mathrm{CDCl}_{3}\right): \delta 27.11(\mathrm{Me}), 52.24$ $(\mathrm{C}(1 \phi)), 55.74(\mathrm{MeO}), 111.02,115.05,119.48,121.50,121.82,133.82,139.48$, 155.00, $161.53(\mathrm{C}(2)), 202.78(\mathrm{C}(2 \notin))$. Anal. Calcd for $\mathrm{C}_{13} \mathrm{H}_{13} \mathrm{NO}_{3}$ : C, 67.52; $\mathrm{H}, 5.67 ; \mathrm{N}, 6.06$. Found: $\mathrm{C}, 67.64 ; \mathrm{H}, 5.75 ; \mathrm{N}, 6.03$.

27b: $7 \%$ yield. Mp 78-79 ${ }^{\circ} \mathrm{C} .{ }^{1} \mathrm{H}$ NMR $\left(400 \mathrm{MHz}, \mathrm{CDCl}_{3}\right): \delta 2.25(\mathrm{~s}$, $3 \mathrm{H}, \mathrm{Me}), 3.89$ (s, $3 \mathrm{H}, \mathrm{MeO}), 5.00$ (s, 2H, H-C(1ф)), 7.00 (d, $1 \mathrm{H}, J=8.8 \mathrm{~Hz}$, $\mathrm{H}-\mathrm{C}(3)), 7.05(\mathrm{~d}, 1 \mathrm{H}, J=2.8 \mathrm{~Hz}, \mathrm{H}-\mathrm{C}(5)), 7.27(\mathrm{dd}, 1 \mathrm{H}, J=9.2,2.8 \mathrm{~Hz}$, $\mathrm{H}-\mathrm{C}(7)), 7.68(\mathrm{~d}, 1 \mathrm{H}, J=9.2 \mathrm{~Hz}, \mathrm{H}-\mathrm{C}(8)), 7.95(\mathrm{~d}, 1 \mathrm{H}, J=8.8 \mathrm{~Hz}, \mathrm{H}-\mathrm{C}(4)) .{ }^{13} \mathrm{C}$ NMR (100 MHz, $\left.\mathrm{CDCl}_{3}\right): \delta 26.42(\mathrm{Me}), 55.51(\mathrm{MeO}), 70.08(\mathrm{C}(1 \phi)), 106.15$, $112.61,121.31,125.97,128.48,138.36,141.29,156.37,159.29$ (C(2)), 205.07 $(\mathrm{C}(2 \phi))$. Anal. Calcd for $\mathrm{C}_{13} \mathrm{H}_{13} \mathrm{NO}_{3}: \mathrm{C}, 67.52 ; \mathrm{H}, 5.67 ; \mathrm{N}, 6.06$. Found: $\mathrm{C}$, $67.50 ; \mathrm{H}, 5.66 ; \mathrm{N}, 6.06$

6-Benzyloxy-1-(2-oxopropyl)quinolin-2(1H)-one (28a) and 6-Benzyloxy-2-(2-oxopropoxy)quinoline (28b)

Prepared from 6-benzyloxyquinolin-2(1H)-one and chloroacetone by the same procedure as described for 13a and 13b. 28a: $71 \%$ yield. Mp 184$185^{\circ} \mathrm{C} .{ }^{1} \mathrm{H}$ NMR $\left(400 \mathrm{MHz}, \mathrm{CDCl}_{3}\right): \delta 2.22(\mathrm{~s}, 3 \mathrm{H}, \mathrm{Me}), 5.09$ (s, $\left.2 \mathrm{H}, \mathrm{CH}_{2} \mathrm{O}\right)$, $5.10(s, 2 \mathrm{H}, \mathrm{H}-\mathrm{C}(1 \phi)), 6.73(\mathrm{~d}, 1 \mathrm{H}, J=9.2 \mathrm{~Hz}, \mathrm{H}-\mathrm{C}(3)), 6.94(\mathrm{~d}, 1 \mathrm{H}, J=9.2$ $\mathrm{Hz}, \mathrm{H}-\mathrm{C}(8)), 7.10(\mathrm{~d}, 1 \mathrm{H}, J=2.8 \mathrm{~Hz}, \mathrm{H}-\mathrm{C}(5)), 7.20(\mathrm{dd}, 1 \mathrm{H}, J=9.2,2.8 \mathrm{~Hz}$, $\mathrm{H}-\mathrm{C}(7)), 7.33-7.45(\mathrm{~m}, 5 \mathrm{H}, \mathrm{ArH}), 7.66(1 \mathrm{H}, \mathrm{d}, J=9.6 \mathrm{~Hz}, \mathrm{H}-\mathrm{C}(4)) .{ }^{13} \mathrm{C} \mathrm{NMR}$ $\left(100 \mathrm{MHz}, \mathrm{CDCl}_{3}\right): \delta 27.39(\mathrm{Me}), 52.44(\mathrm{C}(1 \notin)), 70.80\left(\mathrm{CH}_{2} \mathrm{O}\right), 112.54$, $115.30,120.37,121.70,122.02,127.71,128.45,128.95,134.16,136.67$, 139.75, 154.31, $161.77(\mathrm{C}(2)), 202.98(\mathrm{C}(2 \phi))$. Anal. Calcd for $\mathrm{C}_{19} \mathrm{H}_{17} \mathrm{NO}_{3}: \mathrm{C}$, $74.25 ; \mathrm{H}, 5.58 ; \mathrm{N}, 4.56$. Found: C, 74.16; H, 5.57; N, 4.52.

28b: $10 \%$ yield. Mp $115-116^{\circ} \mathrm{C} .{ }^{1} \mathrm{H}$ NMR $\left(400 \mathrm{MHz}, \mathrm{CDCl}_{3}\right): \delta 2.26(\mathrm{~s}$, $3 \mathrm{H}, \mathrm{Me}), 5.01\left(\mathrm{~s}, 2 \mathrm{H}, \mathrm{CH}_{2} \mathrm{O}\right), 5.15(\mathrm{~s}, 2 \mathrm{H}, \mathrm{H}-\mathrm{C}(1 \phi)), 7.01(\mathrm{~d}, 1 \mathrm{H}, J=8.8 \mathrm{~Hz}$, $\mathrm{H}-\mathrm{C}(3)), 7.14$ (d, $1 \mathrm{H}, J=2.8 \mathrm{~Hz}, \mathrm{H}-\mathrm{C}(5)), 7.33-7.43$ (m, 3H, ArH), 7.36 (dd, $1 \mathrm{H}, J=9.2,2.8 \mathrm{~Hz}, \mathrm{H}-\mathrm{C}(7)), 7.47-7.48(\mathrm{~m}, 2 \mathrm{H}, \mathrm{ArH}), 7.70(\mathrm{~d}, 1 \mathrm{H}, J=9.2$ $\mathrm{Hz}, \mathrm{H}-\mathrm{C}(8)), 7.94(\mathrm{~d}, 1 \mathrm{H}, J=8.8 \mathrm{~Hz}, \mathrm{H}-\mathrm{C}(4)) .{ }^{13} \mathrm{C}$ NMR $\left(100 \mathrm{MHz}, \mathrm{CDCl}_{3}\right)$ : $\delta 26.72(\mathrm{Me}), 70.28(\mathrm{C}(1 \mathrm{c})), 70.56\left(\mathrm{CH}_{2} \mathrm{O}\right), 107.83,112.93,114.14,121.99$, $126.20,127.77,128.34,128.89,136.94,138.64,141.74,155.74,159.63(C(2))$, $205.45(\mathrm{C}(2 \not))$. Anal. Calcd for $\mathrm{C}_{19} \mathrm{H}_{17} \mathrm{NO}_{3}: \mathrm{C}, 74.25 ; \mathrm{H}, 5.58 ; \mathrm{N}, 4.56$. Found: C, 74.44; H, 5.60; N, 4.55 .

6-Chloro-1-(2-oxopropyl)quinolin-2(1H)-one (29a) and 6-Chloro-2-(2oxopropoxy)quinoline (29b)

Prepared from 6-chloroquinolin-2(1H)-one and chloroacetone by the same procedure as described for 13a and 13b. 29a: $85 \%$ yield. Mp $178-179{ }^{\circ} \mathrm{C} .{ }^{1} \mathrm{H}$ NMR (400 MHz, $\left.\mathrm{CDCl}_{3}\right): \delta 2.27(\mathrm{~s}, 3 \mathrm{H}, \mathrm{Me}), 5.10(\mathrm{~s}, 2 \mathrm{H}, \mathrm{H}-\mathrm{C}(1 \phi)), 6.76(\mathrm{~d}$, $1 \mathrm{H}, J=9.6 \mathrm{~Hz}, \mathrm{H}-\mathrm{C}(3)), 6.92(\mathrm{~d}, 1 \mathrm{H}, J=9.2 \mathrm{~Hz}, \mathrm{H}-\mathrm{C}(8)), 7.44$ (dd, $1 \mathrm{H}, J=$ 9.2, $2.0 \mathrm{~Hz}, \mathrm{H}-\mathrm{C}(7)), 7.56(\mathrm{~d}, 1 \mathrm{H}, J=2.0 \mathrm{~Hz}, \mathrm{H}-\mathrm{C}(5)), 7.65(\mathrm{~d}, 1 \mathrm{H}, J=9.6 \mathrm{~Hz}$, $\mathrm{H}-\mathrm{C}(4)) .{ }^{13} \mathrm{C}$ NMR $\left(100 \mathrm{MHz}, \mathrm{CDCl}_{3}\right): \delta 27.22(\mathrm{Me}), 52.07$ (C(1ф)), 115.24, $121.71,122.53,128.01,128.21,130.83,137.90,138.80,161.48(C(2)), 201.79$ $(\mathrm{C}(2 \phi))$. Anal. Calcd for $\mathrm{C}_{12} \mathrm{H}_{10} \mathrm{NO}_{2} \mathrm{Cl}: \mathrm{C}, 61.16 ; \mathrm{H}, 4.28 ; \mathrm{N}, 5.94$. Found: $\mathrm{C}$,
61.13; H, 4.32; N, 6.01

29b: $9 \%$ yield. Mp $108-109{ }^{\circ} \mathrm{C} .{ }^{1} \mathrm{H}$ NMR $\left(400 \mathrm{MHz}, \mathrm{CDCl}_{3}\right): \delta 2.26(\mathrm{~s}$, $3 \mathrm{H}, \mathrm{Me}), 5.03(\mathrm{~s}, 2 \mathrm{H}, \mathrm{H}-\mathrm{C}(1 \phi)), 7.06(\mathrm{~d}, 1 \mathrm{H}, J=8.8 \mathrm{~Hz}, \mathrm{H}-\mathrm{C}(3)), 7.54(\mathrm{dd}, 1 \mathrm{H}$, $J=8.8,2.0 \mathrm{~Hz}, \mathrm{H}-\mathrm{C}(7)), 7.69(\mathrm{~d}, 1 \mathrm{H}, J=8.8 \mathrm{~Hz}, \mathrm{H}-\mathrm{C}(8)), 7.70(\mathrm{~d}, 1 \mathrm{H}, J=2.8$ $\mathrm{Hz}, \mathrm{H}-\mathrm{C}(5)), 7.95$ (d, $1 \mathrm{H}, J=8.8 \mathrm{~Hz}, \mathrm{H}-\mathrm{C}(4)) .{ }^{13} \mathrm{C}$ NMR $\left(100 \mathrm{MHz}, \mathrm{CDCl}_{3}\right)$ : 反 $26.42(\mathrm{Me}), 70.14(\mathrm{C}(1 \notin)), 113.64,126.04,126.25,128.81,129.91,130.35$, 138.42, 144.46, $160.84(\mathrm{C}(2)), 204.39(\mathrm{C}(2 \varnothing))$. Anal. Caled for $\mathrm{C}_{12} \mathrm{H}_{10} \mathrm{NO}_{2} \mathrm{Cl}$ : C, 61.16; H, 4.28; N, 5.94. Found: C, 61.18; H, 4.37; N, 5.93.

7-Methoxy-1-(2-oxopropyl)quinolin-2(1H)-one (30a) and 7-Methoxy2-(2-oxopropoxy)quinoline (30b)

Prepared from 7-methoxyquinolin-2(1H)-one and chloroacetone by the same procedure as described for 13a and 13b. 30a: $84 \%$ yield. Mp $113-114{ }^{\circ} \mathrm{C}$. ${ }^{1} \mathrm{H} \mathrm{NMR}\left(400 \mathrm{MHz}, \mathrm{CDCl}_{3}\right.$ ): $\delta 2.20$ (s, $\left.3 \mathrm{H}, \mathrm{Me}\right), 3.84$ (s, 3H, $\mathrm{MeO}$ ), 5.05 (s, $2 \mathrm{H}$, $\mathrm{H}-\mathrm{C}(1 \notin)), 6.44(\mathrm{~d}, 1 \mathrm{H}, J=2.0 \mathrm{~Hz}, \mathrm{H}-\mathrm{C}(8)), 6.56(\mathrm{~d}, 1 \mathrm{H}, J=9.6 \mathrm{~Hz}, \mathrm{H}-\mathrm{C}(3))$, $6.81(\mathrm{dd}, 1 \mathrm{H}, J=8.8,2.0 \mathrm{~Hz}, \mathrm{H}-\mathrm{C}(6)), 7.48(\mathrm{~d}, 1 \mathrm{H}, J=8.8 \mathrm{~Hz}, \mathrm{H}-\mathrm{C}(5)), 7.66$ $(\mathrm{d}, 1 \mathrm{H}, J=9.6 \mathrm{~Hz}, \mathrm{H}-\mathrm{C}(4)) .{ }^{13} \mathrm{C} \mathrm{NMR}\left(100 \mathrm{MHz}, \mathrm{CDCl}_{3}\right): \delta 27.30(\mathrm{Me}), 52.50$ $(\mathrm{C}(1 \phi)), 55.83(\mathrm{MeO}), 98.87,110.02,115.15,118.16,130.79,140.07,141.20$, 162.29, $162.51(\mathrm{C}(2)), 203.19(\mathrm{C}(2 \notin))$. Anal. Calcd for $\mathrm{C}_{13} \mathrm{H}_{13} \mathrm{NO}_{3}$ : C, 67.52; H, 5.67; N, 6.06. Found: C, 67.44; H, 5.68; N, 6.00.

30b: $8 \%$ yield. Mp $96-97{ }^{\circ} \mathrm{C} .{ }^{1} \mathrm{H}$ NMR $\left(400 \mathrm{MHz}, \mathrm{CDCl}_{3}\right): \delta 2.26(\mathrm{~s}$, $3 \mathrm{H}, \mathrm{Me}), 3.92(\mathrm{~s}, 3 \mathrm{H}, \mathrm{MeO}), 5.01(\mathrm{~s}, 2 \mathrm{H}, \mathrm{H}-\mathrm{C}(1 \phi)), 6.89(\mathrm{~d}, 1 \mathrm{H}, J=8.8 \mathrm{~Hz}$, $\mathrm{H}-\mathrm{C}(3)), 7.04(\mathrm{dd}, 1 \mathrm{H}, J=8.8,2.4 \mathrm{~Hz}, \mathrm{H}-\mathrm{C}(6)), 7.13(\mathrm{~d}, 1 \mathrm{H}, J=2.4 \mathrm{~Hz}$, $\mathrm{H}-\mathrm{C}(8)), 7.60(\mathrm{~d}, 1 \mathrm{H}, J=8.8 \mathrm{~Hz}, \mathrm{H}-\mathrm{C}(5)), 7.96(\mathrm{~d}, 1 \mathrm{H}, J=8.4 \mathrm{~Hz}, \mathrm{H}-\mathrm{C}(4)) .{ }^{13} \mathrm{C}$ NMR (100 MHz, $\left.\mathrm{CDCl}_{3}\right): \delta 26.68(\mathrm{Me}), 55.71(\mathrm{MeO}), 70.40(\mathrm{C}(1 \mathrm{c})), 106.77$, $109.98,116.90,120.44,128.66,139.25,148.06,161.33,161.44$ (C(2)), 205.58 $(\mathrm{C}(2 \phi))$. Anal. Calcd for $\mathrm{C}_{13} \mathrm{H}_{13} \mathrm{NO}_{3}$ : $\mathrm{C}, 67.52 ; \mathrm{H}, 5.67 ; \mathrm{N}, 6.06$. Found: $\mathrm{C}$, $67.20 ; \mathrm{H}, 5.88 ; \mathrm{N}, 5.93$.

7-Benzyloxy-1-(2-oxopropyl)quinolin-2(1H)-one

(31a)

and

\section{7-Benzyloxy-2-(2-oxopropoxy)quinoline (31b)}

Prepared from 7-benzyloxyquinolin-2(1H)-one and chloroacetone by the same procedure as described for 13a and 13b. 31a: $62 \%$ yield. Mp 103-104 ${ }^{\circ} \mathrm{C}$. ${ }^{1} \mathrm{H}$ NMR $\left(400 \mathrm{MHz}, \mathrm{CDCl}_{3}\right): \delta 2.17$ (s, 3H, Me), $5.02\left(\mathrm{~s}, 2 \mathrm{H}, \mathrm{CH}_{2} \mathrm{O}\right), 5.12$ (s, $2 \mathrm{H}, \mathrm{H}-\mathrm{C}(1 \mathrm{c})), 6.51(\mathrm{~d}, 1 \mathrm{H}, J=2.4 \mathrm{~Hz}, \mathrm{H}-\mathrm{C}(8)), 6.58(\mathrm{~d}, 1 \mathrm{H}, J=9.6 \mathrm{~Hz}$, $\mathrm{H}-\mathrm{C}(3)), 6.89$ (dd, $1 \mathrm{H}, J=8.8,2.4 \mathrm{~Hz}, \mathrm{H}-\mathrm{C}(6)), 7.35-7.44$ (m, 5H, ArH), 7.49 $(\mathrm{d}, 1 \mathrm{H}, J=8.8 \mathrm{~Hz}, \mathrm{H}-\mathrm{C}(5)), 7.66(\mathrm{~d}, 1 \mathrm{H}, J=9.6 \mathrm{~Hz}, \mathrm{H}-\mathrm{C}(4)) .{ }^{13} \mathrm{C} \mathrm{NMR}(100$ $\left.\mathrm{MHz}, \mathrm{CDCl}_{3}\right): \delta 27.31(\mathrm{Me}), 52.49(\mathrm{C}(1 \phi)), 70.68\left(\mathrm{CH}_{2} \mathrm{O}\right), 99.95,110.81$, $115.32,118.30,127.78,128.61,129.02,130.79,136.21,140.05,141.14$ 161.35, $162.50(\mathrm{C}(2)), 202.99(\mathrm{C}(2 \notin))$. Anal. Calcd for $\mathrm{C}_{19} \mathrm{H}_{17} \mathrm{NO}_{3}$ : C, 74.25; H, 5.58; N, 4.56. Found: C, 74.27; H, 5.59; N, 4.54.

31b: $8 \%$ yield. Mp 133-134 ${ }^{\circ} \mathrm{C} .{ }^{1} \mathrm{H}$ NMR $\left(400 \mathrm{MHz}, \mathrm{CDCl}_{3}\right): \delta 2.26$ (s, 3H, Me), $5.01\left(\mathrm{~s}, 2 \mathrm{H}, \mathrm{CH}_{2} \mathrm{O}\right), 5.18(\mathrm{~s}, 2 \mathrm{H}, \mathrm{H}-\mathrm{C}(1 \phi)), 6.90(\mathrm{~d}, 1 \mathrm{H}, J=8.4$ $\mathrm{Hz}, \mathrm{H}-\mathrm{C}(3)), 7.13(\mathrm{dd}, 1 \mathrm{H}, J=8.8,2.4 \mathrm{~Hz}, \mathrm{H}-\mathrm{C}(6)), 7.24(\mathrm{~d}, 1 \mathrm{H}, J=2.4 \mathrm{~Hz}$, $\mathrm{H}-\mathrm{C}(8)), 7.34-7.38(\mathrm{~m}, 1 \mathrm{H}, \mathrm{ArH}), 7.40-7.44(\mathrm{~m}, 2 \mathrm{H}, \mathrm{ArH}), 7.48-7.51(\mathrm{~m}, 2 \mathrm{H}$, $\mathrm{ArH}), 7.63(\mathrm{~d}, 1 \mathrm{H}, J=8.8 \mathrm{~Hz}, \mathrm{H}-\mathrm{C}(5)), 7.97(\mathrm{~d}, 1 \mathrm{H}, J=8.4 \mathrm{~Hz}, \mathrm{H}-\mathrm{C}(4)) .{ }^{13} \mathrm{C}$ NMR $\left(100 \mathrm{MHz}, \mathrm{CDCl}_{3}\right): \delta 26.72(\mathrm{Me}), 70.39(\mathrm{C}(1 \phi)), 70.47\left(\mathrm{CH}_{2} \mathrm{O}\right), 107.90$, $110.12,117.25,120.59,127.90,128.38,128.76,128.89,136.75,139.29$, 147.96, 160.48, $161.43(\mathrm{C}(2)), 205.54(\mathrm{C}(2 \notin))$. Anal. Calcd for $\mathrm{C}_{19} \mathrm{H}_{17} \mathrm{NO}_{3}$ : C, 74.25; H, 5.58; N, 4.56. Found: C, 74.24; H, 5.57; N, 4.53.

7-Chloro-1-(2-oxopropyl)quinolin-2(1H)-one (32a) and 7-Chloro-2-(2oxopropoxy)quinoline (32b)

Prepared from 7-chloroquinolin-2(1H)-one and chloroacetone by the same procedure as described for 13a and 13b. 32a: $76 \%$ yield. $\mathrm{Mp} 158-159^{\circ} \mathrm{C} .{ }^{1} \mathrm{H}$ NMR (400 MHz, DMSO- $d$ ): $\delta 2.31$ (s, 3H, Me), $5.23(\mathrm{~s}, 2 \mathrm{H}, \mathrm{H}-\mathrm{C}(1 \phi)), 6.65$ $(\mathrm{d}, 1 \mathrm{H}, J=9.2 \mathrm{~Hz}, \mathrm{H}-\mathrm{C}(3)), 7.31$ (dd, $1 \mathrm{H}, J=8.4,1.6 \mathrm{~Hz}, \mathrm{H}-\mathrm{C}(6)), 7.51$ (d, $1 \mathrm{H}, J=1.6 \mathrm{~Hz}, \mathrm{H}-\mathrm{C}(8)), 7.77(\mathrm{~d}, 1 \mathrm{H}, J=8.4 \mathrm{~Hz}, \mathrm{H}-\mathrm{C}(5)), 7.98(\mathrm{~d}, 1 \mathrm{H}, J=9.6$ $\mathrm{Hz}, \mathrm{H}-\mathrm{C}(4)) .{ }^{13} \mathrm{C}$ NMR (100 MHz, DMSO- $\left.d_{6}\right): \delta 28.10(\mathrm{Me}), 52.34(\mathrm{C}(1 \phi))$, $115.06,119.51,121.46,122.98,131.22,136.41,140.27,141.09,161.56(\mathrm{C}(2))$, $202.86(\mathrm{C}(2 \notin))$. Anal. Calcd for $\mathrm{C}_{12} \mathrm{H}_{10} \mathrm{NO}_{2} \mathrm{Cl}: \mathrm{C}, 61.16 ; \mathrm{H}, 4.28 ; \mathrm{N}, 5.94$. Found: C, $61.09 ; \mathrm{H}, 4.25 ; \mathrm{N}, 5.96$.

32b: $8 \%$ yield. Mp $93-94{ }^{\circ} \mathrm{C} .{ }^{1} \mathrm{H}$ NMR $(400 \mathrm{MHz}, \mathrm{CDCl})$ ) $\delta 2.27(\mathrm{~s}, 3 \mathrm{H}$, Me), 5.04 (s, 2H, H-C(1ф)), 7.03 (d, $1 \mathrm{H}, J=8.8 \mathrm{~Hz}, \mathrm{H}-\mathrm{C}(3)), 7.35$ (dd, $1 \mathrm{H}, J$ $=8.4,2.0 \mathrm{~Hz}, \mathrm{H}-\mathrm{C}(6)), 7.65(\mathrm{~d}, 1 \mathrm{H}, J=8.4 \mathrm{~Hz}, \mathrm{H}-\mathrm{C}(5)), 7.79(\mathrm{~d}, 1 \mathrm{H}, J=1.6$ $\mathrm{Hz}, \mathrm{H}-\mathrm{C}(8)), 8.12$ (d, $1 \mathrm{H}, J=8.8 \mathrm{~Hz}, \mathrm{H}-\mathrm{C}(4)) .{ }^{13} \mathrm{C}$ NMR $\left(100 \mathrm{MHz}, \mathrm{CDCl}_{3}\right.$ ): $\delta 26.67(\mathrm{Me}), 70.62(\mathrm{C}(1 \mathrm{c})), 113.02,123.95,125.69,126.51,128.84,135.94$ 139.55, 146.51, $161.53(\mathrm{C}(2)), 204.19(\mathrm{C}(2 \varnothing))$. Anal. Caled for $\mathrm{C}_{12} \mathrm{H}_{10} \mathrm{NO}_{2} \mathrm{Cl}$ : C, 61.16; H, 4.28; N, 5.94. Found: C, 61.19; H, 4.28; N, 5.93.

\section{8-Methoxy-2-(2-oxopropoxy)quinoline (34b)}

Prepared from 8-methoxyquinolin-2 $(1 H)$-one and chloroacetone by the same procedure as described for 13a and $13 \mathrm{~b}$. $67 \%$ yield. Mp $55-56{ }^{\circ} \mathrm{C} .{ }^{1} \mathrm{H}$ NMR (400 MHz, $\left.\mathrm{CDCl}_{3}\right): \delta 2.33$ (s, 3H, Me), 4.01 (s, 3H, MeO), 5.09 (s, $2 \mathrm{H}$, $\mathrm{H}-\mathrm{C}(1 \mathrm{c})), 7.03-7.05(\mathrm{~m}, 1 \mathrm{H}, \mathrm{ArH}), 7.07(\mathrm{~d}, 1 \mathrm{H}, J=8.8 \mathrm{~Hz}, \mathrm{H}-\mathrm{C}(3)), 7.31-7.34$ (m, 2H, ArH), $8.02(\mathrm{~d}, 1 \mathrm{H}, J=8.8 \mathrm{~Hz}, \mathrm{H}-\mathrm{C}(4)) .{ }^{13} \mathrm{C} \mathrm{NMR}\left(100 \mathrm{MHz}, \mathrm{CDCl}_{3}\right)$ : $\delta 26.84(\mathrm{Me}), 56.20(\mathrm{MeO}), 69.95(\mathrm{C}(1 \phi)), 109.38,112.88,119.60,124.44$, 
126.51, 137.40, 139.50, 154.17, $159.96(\mathrm{C}(2)), 204.66(\mathrm{C}(2 \varnothing))$. Anal. Calcd for $\mathrm{C}_{13} \mathrm{H}_{13} \mathrm{NO}_{3}$ : C, 67.52; H, 5.67; N, 6.06. Found: C, 67.51; H, 5.73; N, 5.90.

8-Benzyloxy-2-(2-oxopropoxy)quinoline (35b)

Prepared from 8-benzyloxyquinolin-2(1H)-one and chloroacetone by the same procedure as described for $13 \mathbf{a}$ and $\mathbf{1 3 b}$. $75 \%$ yield. Mp $56-57{ }^{\circ} \mathrm{C} .{ }^{1} \mathrm{H}$ NMR (400 MHz, CDCl $): \delta 2.15(\mathrm{~s}, 3 \mathrm{H}, \mathrm{Me}), 5.00\left(\mathrm{~s}, 2 \mathrm{H}, \mathrm{CH}_{2} \mathrm{O}\right), 5.24(\mathrm{~s}, 2 \mathrm{H}$ $\mathrm{H}-\mathrm{C}(1 \varnothing)), 7.06(\mathrm{~d}, 1 \mathrm{H}, J=8.8 \mathrm{~Hz}, \mathrm{H}-\mathrm{C}(3)), 7.09-7.11(\mathrm{~m}, 1 \mathrm{H}, \mathrm{ArH}), 7.25-7.42$ (m, 5H, ArH), 7.52-7.54 (m, 2H, ArH), 8.02 (d, $1 \mathrm{H}, J=8.8 \mathrm{~Hz}, \mathrm{H}-\mathrm{C}(4)) .{ }^{13} \mathrm{C}$ NMR (100 MHz, $\left.\mathrm{CDCl}_{3}\right): \delta 26.52(\mathrm{Me}), 69.84(\mathrm{C}(1 \varnothing)), 71.07\left(\mathrm{CH}_{2} \mathrm{O}\right), 111.76$, $112.72,120.08,124.31,126.60,127.49,127.86,128.45,137.20,137.83$ 139.39, 153.27, $159.81(\mathrm{C}(2)), 204.48(\mathrm{C}(2 \varnothing))$. Anal. Calcd for $\mathrm{C}_{19} \mathrm{H}_{17} \mathrm{NO}_{3}: \mathrm{C}$, 74.25; H, 5.58; N, 4.56. Found: C, 74.27; H, 5.71; N, 4.38.

8-Chloro-2-(2-oxopropoxy)quinoline (36b)

Prepared from 8-chloroquinolin-2(1H)-one and chloroacetone by the same procedure as described for 13a and 13b. $89 \%$ yield. Mp 99-100 ${ }^{\circ} \mathrm{C} .{ }^{1} \mathrm{H}$ NMR (400 MHz, $\mathrm{CDCl}_{3}$ ): $\delta 2.33$ (s, 3H, Me), 5.09 (s, 2H, H-C(1ф)), 7.10 (d, 1H, J= $8.8 \mathrm{~Hz}, \mathrm{H}-\mathrm{C}(3)), 7.30(\mathrm{dd}, 1 \mathrm{H}, J=7.6,7.6 \mathrm{~Hz}, \mathrm{H}-\mathrm{C}(6)), 7.64$ (dd, $1 \mathrm{H}, J=8.0$, $1.2 \mathrm{~Hz}, \mathrm{H}-\mathrm{C}(7)), 7.72$ (dd, $1 \mathrm{H}, J=7.6,1.2 \mathrm{~Hz}, \mathrm{H}-\mathrm{C}(5)), 8.05$ (d, $1 \mathrm{H}, J=8.8 \mathrm{~Hz}$, $\mathrm{H}-\mathrm{C}(4)) .{ }^{13} \mathrm{C} \mathrm{NMR}\left(100 \mathrm{MHz}, \mathrm{CDCl}_{3}\right): \delta 26.72(\mathrm{Me}), 70.16(\mathrm{C}(1 \varnothing)), 113.35$, $124.35,126.37,126.58,129.86,131.44,139.75,142.33,160.89$ (C(2)), 203.87 $(\mathrm{C}(2 \varnothing))$. Anal. Calcd for $\mathrm{C}_{12} \mathrm{H}_{10} \mathrm{NO}_{2} \mathrm{Cl}: \mathrm{C}, 61.16 ; \mathrm{H}, 4.28 ; \mathrm{N}, 5.94$. Found: $\mathrm{C}$, $61.17 ; \mathrm{H}, 4.23 ; \mathrm{N}, 5.98$.

\section{REFERENCES}

1. M. Tominaga, H. Tone, K. Nakagawa, K. Takada, Y. Hoshino, K. Watanabe, Chem. Pharm. Bull. 29, 2166, (1981).

2. T. Nishi, K. Yamamoto, T. Shimizu, T. Kanbe, Y. Kimura, K. Nakagawa, Chem. Pharm. Bull. 31, 798, (1983).

3. P. Cheng, Q. Zhang, Y.B. Ma, Z.Y. Jiang, X.M. Zhang, F.X. Zhang, J.J Chen, Bioorg. Med. Chem. Lett. 18, 3787, (2008).

4. C.D. Beadle, J. Boot, N.P. Camp, N. Dezutter, J. Findlay, L. Hayhurst, J.J. Masters, R. Penariol, M.W. Walter, Bioorg. Med. Chem. Lett. 15, 4432, (2005).

5. J.T. Kuethe, A. Wong, C. Qu, J. Smitrovich, I.W. Davies, D.L. Hughes, J. Org. Chem. 70, 2555, (2005).
6. P. Hewawasam, W. Fan, D.A. Cook, K.S. Newberry, C.G. Boissard, V.K. Gribkoff, J. Starrett, N.J. Lodge, Bioorg. Med. Chem. Lett. 14, 4479, (2004).

7. T. Fujioka, S. Teramoto, T. Mori, T. Hosokawa, T. Sumida, M. Tominaga, Y. Yabuuchi, J. Med. Chem. 35, 3607, (1992).

8. B.J. Lipworth, A. Grove, Br. J. Clin. Pharm. 43, 9, (1997).

9. C.C. Tzeng, T.C. Wang, Y.L. Chen, C.J. Wang, Y.L. Chang, C.M. Teng, Helv. Chim. Acta. 80, 1161, (1007).

10. I.L. Chen, Y.L. Chen, T.C. Wang, C.C. Tzeng, Heterocycles 60, 131, (2003).

11. T.C. Wang, Y.L. Chen, C.C. Tzeng, S.S. Liou, W.F. Tzeng, Y.L. Chang, C.M. Teng, Helv. Chim. Acta. 81, 1038, (1998).

12. S.S. Liou, Y.L. Zhao, Y.L. Chang, C.M. Teng, C.C. Tzeng, Chem. Pharm. Bull. 45, 1777, (1997).

13. Y.L. Chen, T.C. Wang, K.C. Fang, N.C. Chang, C.C. Tzeng, Heterocycles 50, 453, (1999).

14. C.C. Tzeng, K.H. Lee, T.C. Wang, C.H. Han, Y.L. Chen, Pharmaceut. Res. 17, 715, (2000).

15. T. Sato, K. Yoshimatsu, J. Otera, Synlett 845, (1995).

16. H. Liu, S.-B. Ko, H. Josien, D. P. Curran, Tetrahedron Lett. 36, 8917, (1995).

17. Y. L. Janin, C. Huel, G. Flad, S. Thirot, Eur. J. Org. Chem. 1763, (2002).

18. G. Hopkins, J.P. Jonak, H.J. Minnemeyer, H. Tieckelmann, J. Org. Chem. 32, 4040, (1967).

19. D. L. Comins, G. Jianhua, Tetrahedron Lett. 35, 2819, (1994).

20. M. C. Torhan, N. P. Peet, J. D. Williams, Tetrahedron Letters 54, 3926, (2013)

21. M. Hadjeri, A.-M. Mariotte, A. Boumendjel, Chem. Pharm. Bull. 49 (10) 1352, (2001).

22. P. Depreux, G. Bethegnies, Synth. Commun. 23(8), 1189 (1993).

23. M. Hedayatullah, J. Heterocycl. Chem. 18, 339 (1981).

24. T. Yamada, M. Ohki, Synthesis 631 (1981).

25. C. G. Dave, K. J. Patel, J. Heterocycl Chem., 51, 943 (2014).

26. Z.X. Guo, A.N. Cammidge, A. McKillop, D.C. Horwell, Tetrahedron Lett. 40, 6999, (1999). 